\title{
INTERACTION OF LONGITUDINAL WAVES WITH TRANSVERSE WAVES IN DISPERSIVE NONLINEAR ELASTIC MEDIA. I*
}

\author{
BY \\ Y. M. CHEN \\ State University of New York at Stony Brook
}

I. Introduction. Interest in elastic wave propagation in nonlinear media has grown considerably in recent years. This is probably due to the increasing number of sophisticated applications in engineering and science where the simple linear theory breaks down. Until now, most of the existing literature on waves in nonlinear solids (see [1] and its references) considers only the propagation of one-dimensional longitudinal or transverse waves. They are solved either by the method of characteristics for a system of two reducible first-order hyperbolic partial differential equations with two independent variables, or by related methods.

In this paper the simultaneous propagation of periodic plane harmonic longitudinal and transverse waves (with angular frequency $\omega$ known) in isotropic nonlinear dispersive elastic media of infinite extent is studied. The dispersion comes from the equivalent body forces which are assumed to be functions of the displacement vector $\boldsymbol{u}$ and its various partial derivatives. This kind of mathematical model can be realized approximately for wave propagation in a medium composed of a soft nonlinear elastic base material with stiff reinforcing structures imbedded in the base. Our analysis is based upon a generalized version of the iteration method previously employed to construct solutions of the eigenvalue problem of simpler nonlinear partial differential operators [2,3]. One interesting feature of this method is that it yields no secular terms.

In Sec. 2, the equations of motion for wave propagation in nonlinear dispersive elastic media are derived in the third approximation. In Sec. 3, the problem of simultaneous propagation of periodic plane harmonic longitudinal and transverse waves in an infinite isotropic nonlinear dispersive elastic medium is analyzed by using the above-mentioned iteration scheme. General formulae for the iterated displacement vector and propagation constants are given; the first iteration is carried out explicitly in Sec. 4. Effects of the equivalent body forces and the nonlinearity of the medium on the interaction between longitudinal and transverse waves and on their respective dispersion relations are discussed in the last section.

2. Basic equations. In nonlinear elastic media, the strain tensor can be expressed as

$$
u_{i j}=\frac{1}{2}\left(\frac{\partial u_{i}}{\partial x_{i}}+\frac{\partial u_{i}}{\partial x_{i}}+\frac{\partial u_{k}}{\partial x_{i}} \frac{\partial u_{k}}{\partial x_{j}}\right), \quad i, j, k=1,2,3
$$

* Received December 10, 1969; revised version received March 19, 1970. This work was supported in part by the National Science Foundation under Grant GK-2756. 
where $u_{i}$ and $x_{i}$ are the $i$ th components of the displacement vector $u$ and position vector $x$, respectively. If thermodynamic effects are negligible, the elastic energy of an isotropic body in the third approximation has the general form [4]

$$
\mathcal{E}=\mu u_{i j}^{2}+\left(\frac{1}{2} K-\frac{1}{3} \mu\right) u_{k k}^{2}+\frac{1}{3} A u_{i j} u_{i k} u_{j k}+B u_{i j}^{2} u_{k k}+\frac{1}{3} C u_{k k}^{3},
$$

where

$$
K=\lambda+\frac{2}{3} \mu
$$

is the modulus of hydrostatic compression, $\lambda, \mu$ are Lamé coefficients, and $A, B, C$ are parameters which characterize the nonlinear elastic media. By substituting (2.1) into (2.2) and keeping the terms of proper order, we obtain a more explicit form of the elastic energy:

$$
\begin{aligned}
\varepsilon=\frac{1}{4} \mu\left(\frac{\partial u_{i}}{\partial x_{i}}+\frac{\partial u_{i}}{\partial x_{i}}\right)^{2}+\left(\frac{1}{2} K\right. & \left.-\frac{1}{3} \mu\right)\left(\frac{\partial u_{k}}{\partial x_{k}}\right)^{2}+\left(\mu+\frac{1}{4} A\right) \frac{\partial u_{i}}{\partial x_{i}} \frac{\partial u_{k}}{\partial x_{i}} \frac{\partial u_{k}}{\partial x_{i}} \\
& +\left(\frac{1}{2} B+\frac{1}{2} K-\frac{1}{3} \mu\right) \frac{\partial u_{k}}{\partial x_{k}}\left(\frac{\partial u_{i}}{\partial x_{i}}\right)^{2}+\frac{1}{12} A \frac{\partial u_{i}}{\partial x_{i}} \frac{\partial u_{i}}{\partial x_{k}} \frac{\partial u_{k}}{\partial x_{i}} \\
& +\frac{1}{2} B \frac{\partial u_{i}}{\partial x_{i}} \frac{\partial u_{i}}{\partial x_{i}} \frac{\partial u_{k}}{\partial x_{k}}+\frac{1}{3} C\left(\frac{\partial u_{k}}{\partial x_{k}}\right)^{3}
\end{aligned}
$$

Once the expression of elastic energy is known, the stress tensor $\sigma_{1 ;}$ can be derived from the relation

$$
\sigma_{i j}=\partial \mathcal{E} / \partial\left(\frac{\partial u_{i}}{\partial x_{i}}\right)
$$

Then the equations of motion are

$$
\rho \partial^{2} u_{i} / \partial t^{2}=\partial \sigma_{i j} / \partial x_{i}+F_{i},
$$

where $F_{i}$ is the $i$ th component of a body force $F$ and $\rho$ is the mass density. It would be too cumbersome to write out the corresponding equations of motion in their explicit forms. However, they will be explicitly written out for a special case in the next section.

In this paper, we shall only consider the special type of nonlinear dispersive elastic media in which $F$ is a function of $u$ and its various derivatives. Thus

$$
F_{i}=\Gamma_{i} u_{i}+P_{i}\left(u, \frac{\partial u}{\partial x_{i}}, \frac{\partial u}{\partial t}, \cdots\right), \quad \Gamma_{i} \neq 0,
$$

where $\Gamma_{i} u_{i}$ is the only linear part of $F_{i}$. This type of mathematical model can be realized for physical problems in the following manner. Consider the case in which the elastic medium is composed of a soft nonlinear elastic base material and stiff reinforcing structures imbedded in the base. On the one hand, one can solve the problem of wave propagation in this type of media very crudely by using the method of averaging or smoothing. There the averaged or effective elastic constants are used and the interaction of the soft nonlinear elastic material with the stiff reinforcing structure is completely eliminated by the averaging process. On the other hand, one can try to solve this boundary value problem exactly. However, this approach is much too complex to yield any useful results. Nevertheless, a combination of these two approaches can be used, if one understands what the stiff reinforcing structures really do to the base material. In reality 
these reinforcing structures mainly serve as constraining forces on the motion of the base material. Thus, in forming a better model for the problem, the effects of the reinforcing structure on the base material can be incorporated into the equations of motion for the base material alone as equivalent body forces $F$. Because these equivalent body forces come into effect only if there is any displacement of the base material from its equilibrium position, $F$ must be a function of $u$ and its various partial derivatives.

3. General formulation. For plane harmonic elastic waves propagating in the $x_{1}$ direction, the equations governing the displacement field can be deduced from (2.4)(2.7):

$$
\begin{array}{r}
\left(\frac{4}{3} \mu+K\right) \frac{\partial^{2} u_{1}}{\partial x_{1}^{2}}-\rho \frac{\partial^{2} u_{1}}{\partial t^{2}}+\alpha_{1} \frac{\partial u_{1}}{\partial x_{1}} \frac{\partial^{2} u_{1}}{\partial x_{1}^{2}}+\alpha_{5} \frac{\partial u_{2}}{\partial x_{1}} \frac{\partial^{2} u_{2}}{\partial x_{1}^{2}}+\Gamma_{2} u_{1}+P_{1}\left(u_{1}, u_{2}, \cdots\right)=0 \\
\mu \frac{\partial^{2} u_{2}}{\partial x_{1}^{2}}-\rho \frac{\partial^{2} u_{2}}{\partial t^{2}}+\alpha_{5}\left(\frac{\partial u_{2}}{\partial x_{1}} \frac{\partial^{2} u_{1}}{\partial x_{1}^{2}}+\frac{\partial u_{1}}{\partial x_{1}} \frac{\partial^{2} u_{2}}{\partial x_{1}^{2}}\right)+\Gamma_{2} u_{2}+P_{2}\left(u_{1}, u_{2}, \cdots\right)=0
\end{array}
$$

where

$$
\begin{aligned}
& \alpha_{1}=4 \mu+3 K+2 A+6 B+2 C, \\
& \alpha_{5}=\frac{4}{3} \mu+K+\frac{1}{2} A+B .
\end{aligned}
$$

$P_{1}(0,0, \cdots)=P_{2}(0,0, \cdots)=0, \Gamma_{1}>0$ and $\Gamma_{2}>0$. For simplicity, $\Gamma_{1}$ and $\Gamma_{2}$ are assumed to be constants. If we seek a solution of (3.1) which is periodic in both $x_{1}$ and $t$, it is convenient to introduce the new variables:

$$
\begin{aligned}
& x=k_{l} x_{1}, \\
& y=k_{t} x_{1}, \\
& \tau=\omega t,
\end{aligned}
$$

where the angular frequency $\omega$ is regarded as given and $k_{l}$ and $k_{t}$, the propagation constants of the longitudinal and transverse waves respectively, are to be determined. Then the system (3.1) in the new variables takes the form

$$
\begin{gathered}
k_{\imath}^{2} \frac{\partial^{2} u}{\partial x^{2}}-\frac{\omega^{2}}{c_{l}^{2}} \frac{\partial^{2} u}{\partial \tau^{2}}+\gamma_{\imath} u+\beta_{1} k_{l}^{3} \frac{\partial u}{\partial x} \frac{\partial^{2} u}{\partial x^{2}}+\beta_{2} k_{l}^{3} \frac{\partial v}{\partial x} \frac{\partial^{2} v}{\partial x^{2}}+p_{1}(u, v, \cdots)=\mathbf{0}, \\
k_{\imath}^{2} \frac{\partial^{2} v}{\partial y^{2}}-\frac{\omega^{2}}{c_{\imath}^{2}} \frac{\partial^{2} v}{\partial \tau^{2}}+\gamma_{\imath} v+\beta_{3} k_{\imath}^{3}\left(\frac{\partial v}{\partial y} \frac{\partial^{2} u}{\partial y^{2}}+\frac{\partial u}{\partial y} \frac{\partial^{2} v}{\partial y^{2}}\right)+p_{2}(u, v, \cdots)=0, \\
u(x+2 \pi, \tau+2 \pi)=u(x, \tau), \\
v(y+2 \pi, \tau+2 \pi)=v(y, \tau),
\end{gathered}
$$

where

$$
\begin{aligned}
& c_{\ell}=\left[\left(\frac{4}{3} \mu+K\right) \rho^{-1}\right]^{1 / 2}, \quad \text { (longitudinal sound velocity) } \\
& c_{\mathrm{t}}=\left(\mu \rho^{-1}\right)^{1 / 2}, \quad \text { (transverse sound velocity), } \\
& \gamma_{z}=\Gamma_{1}\left(\frac{4}{3} \mu+K\right)^{-1} \text {, } \\
& \gamma_{\ell}=\Gamma_{2} \mu^{-1} \text {, }
\end{aligned}
$$




$$
\begin{aligned}
p_{1} & =P_{1}\left(\frac{4}{3} \mu+K\right)^{-1}, \\
p_{2} & =P_{2} \mu^{-1} \\
\beta_{1} & =\alpha_{1}\left(\frac{4}{3} \mu+K\right)^{-1}, \\
\beta_{2} & =\alpha_{5}\left(\frac{4}{3} \mu+K\right)^{-1}, \\
\beta_{3} & =\alpha_{5} \mu^{-1} \\
u & =u_{1}, \\
v & =u_{2} .
\end{aligned}
$$

Note that $u=v=0$ is a trivial solution of (3.7)-(3.18).

Finding the values $k_{l}$ and $k_{\imath}$ such that the homogeneous system (3.7)-(3.9) has a nontrivial solution $(u, v)$ is a nonlinear eigenvalue problem. Hence, the iteration method developed in [2], [3] can be used here. Let sequences $\left\{\tilde{u}_{n}\right\},\left\{\tilde{v}_{n}\right\},\left\{k_{l_{n}}\right\}$ and $\left\{k_{t n}\right\}$ be the sequences of $u, v, k_{\imath}$ and $k_{t}$ respectively.

Let

$$
\begin{aligned}
& U_{n}=\tilde{u}_{n}-\tilde{u}_{n-1}, \\
& V_{n}=\tilde{v}_{n}-\tilde{v}_{n-1}, \\
& \sigma_{n}=k_{t n} k_{n n}^{-1}, \quad n=0,1,2,3, \cdots,
\end{aligned}
$$

such that

$$
a_{-1}=u_{-1}=\tilde{v}_{-1}=v_{-1}=0 .
$$

Then the modified Newton's iteration method [2] gives

$$
\begin{aligned}
& k_{l 0}^{2} \frac{\partial^{2} U_{n}}{\partial x^{2}}-\frac{\omega^{2}}{c_{l}^{2}} \frac{\partial^{2} U_{n}}{\partial \tau^{2}}+\gamma_{l} U_{n}=-k_{l n}^{2} \frac{\partial^{2} \tilde{u}_{n-1}}{\partial x^{2}}+\frac{\omega^{2}}{c_{l}^{2}} \frac{\partial^{2} \tilde{u}_{n-1}}{\partial \tau^{2}}-\gamma_{\imath} \tilde{u}_{n-1}-\beta_{1} k_{l n}^{3} \frac{\partial \tilde{u}_{n-1}}{\partial x} \frac{\partial^{2} \tilde{u}_{n-1}}{\partial x^{2}} \\
&-\beta_{2} k_{l n}^{3} \frac{\partial \tilde{c}_{n-1}}{\partial x} \frac{\partial^{2} \tilde{v}_{n-1}}{\partial x^{2}}-p_{1}\left(\tilde{u}_{n-1}, \tilde{v}_{n-1}, \cdots\right) \equiv X_{n} \\
& k_{i 0}^{2} \frac{\partial^{2} V_{n}}{\partial y^{2}}-\frac{\omega^{2}}{c_{l}^{2}} \frac{\partial^{2} V_{n}}{\partial \tau^{2}}+\gamma_{t} V_{n}=-k_{t n}^{2} \frac{\partial^{2} \tilde{v}_{n-1}}{\partial y^{2}}+\frac{\omega^{2}}{c_{t}^{2}} \frac{\partial^{2} \tilde{v}_{n-1}}{\partial \tau^{2}}-\gamma_{t} v_{n-1}-p_{2}\left(\tilde{u}_{n-1}, \tilde{v}_{n-1}, \cdots\right) \\
& \quad-\beta_{3} k_{t n}^{3}\left(\frac{\partial \tilde{v}_{n-1}}{\partial y} \frac{\partial^{2} \tilde{u}_{n-1}}{\partial y^{2}}+\frac{\partial \tilde{u}_{n-1}}{\partial y} \frac{\partial^{2} \tilde{v}_{n-1}}{\partial y^{2}}\right) \equiv Y_{n} \\
& U_{n}(x+2 \pi, \tau+2 \pi)=U_{n}(x, \tau), \\
& V_{n}(x+2 \pi, \tau+2 \pi)=V_{n}(x, \tau), \\
& \tilde{u}_{n}(x, \tau)=\tilde{u}_{n}\left(\sigma_{n}^{-1} y, \tau\right), \\
& \tilde{v}_{n}(y, \tau)= \tilde{v}_{n}\left(\sigma_{n} x, \tau\right), \quad n=0,1,2,3, \cdots
\end{aligned}
$$

From (3.24)-(3.28), the initial approximation $\left(U_{0}, V_{0}\right)$ satisfies

$$
k_{l 0}^{2} \frac{\partial^{2} U_{0}}{\partial x^{2}}-\frac{\omega^{2}}{c_{l}^{2}} \frac{\partial^{2} U_{0}}{\partial \tau^{2}}+\gamma_{l} U_{0}=0,
$$




$$
\begin{aligned}
& k_{\imath 0}^{2} \frac{\partial^{2} V_{0}}{\partial y^{2}}-\frac{\omega^{2}}{c_{t}^{2}} \frac{\partial^{2} V_{0}}{\partial \tau^{2}}+\gamma_{t} V_{0}=0, \\
& U_{0}(x+2 \pi, \tau+2 \pi)=U_{0}(x, \tau), \\
& V_{0}(y+2 \pi, \tau+2 \pi)=V_{0}(y, \tau) .
\end{aligned}
$$

A propagating wave solution of (3.31)-(3.34) is

$$
\begin{aligned}
& U_{0}=u_{0}=\epsilon \cos (x-\tau), \\
& V_{0}=v_{0}=\delta \cos (y-\tau+\varphi),
\end{aligned}
$$

provided

$$
\begin{aligned}
& k_{l 0}^{2}=\omega^{2} / c_{l}^{2}+\gamma_{l}, \\
& k_{t 0}^{2}=\omega^{2} / c_{t}^{2}+\gamma_{l},
\end{aligned}
$$

where $\epsilon$ and $\delta$ are amplitudes and $\varphi$ is the phase difference between $U_{0}$ and $V_{0}$. Note that if $\gamma_{t}=\gamma_{t}=0$, then $\cos \zeta(x-\tau)$ and $\cos \eta(y-\tau)$ for any constants $\zeta$ and $\eta$ still satisfy the respective equations (3.37) and (3.38). In other words, both $k_{t 0}^{2}$ and $k_{t 0}^{2}$, the eigenvalues of the linearized problem, have infinite multiplicity. This often implies the nonexistence of periodic solutions [5] which leads to the appearance of shock waves.

Since nontrivial solutions to the system (3.25)-(3.28) exist, the necessary condition for the existence of solutions to the system (3.25)-(3.28) is that the right sides of (3.25) and (3.26) be orthogonal to $U_{0}$ and $V_{0}$. These orthogonality conditions give the equations for $\left\{k_{l_{n}}\right\}$ and $\left\{k_{t_{n}}\right\}$

$$
\begin{aligned}
& \int_{0}^{2 \pi} \int_{0}^{2 \pi} U_{0} X_{n} d x d \tau=0, \\
& \int_{0}^{2 \pi} \int_{0}^{2 \pi} V_{0} Y_{n} d y d \tau=0, \quad n=1,2,3, \cdots .
\end{aligned}
$$

Furthermore, $U_{n}$ and $V_{n}$ can be made unique by requiring

$$
\begin{aligned}
& \int_{0}^{2 \pi} \int_{0}^{2 \pi} U_{0} U_{n} d x d \tau=0 \\
& \int_{0}^{2 \pi} \int_{0}^{2 \pi} V_{0} V_{n} d y d \tau=0, \quad n=1,2,3, \cdots .
\end{aligned}
$$

The generalized Green's functions for (3.25)-(3.28) can be derived as

$$
\begin{aligned}
G_{a}\left(z, \tau ; z^{\prime}, \tau^{\prime}\right)=\frac{-1}{\pi^{2}} & \sum_{\substack{i=0 \\
(i, i)=(1,1)}}^{\infty} \sum_{\substack{i=0 \\
\left(1,0 i^{2}\right.}}^{\infty} \frac{\epsilon_{i} \epsilon_{j}}{\omega^{2} c_{a}^{-2} j^{2}-\gamma_{a}} \\
& \cdot\left(\cos i z^{\prime} \cos i z \cos j \tau^{\prime} \cos j \tau+\sin i z^{\prime} \sin i z \cos j \tau^{\prime} \cos j \tau\right. \\
& \left.+\cos i z^{\prime} \cos i z \sin j \tau^{\prime} \sin j \tau+\sin i z^{\prime} \sin i z \sin j \tau^{\prime} \sin j \tau\right),
\end{aligned}
$$

where $\epsilon_{0}=\frac{1}{2}, \quad \epsilon_{1}=\epsilon_{2}=\epsilon_{3}=\cdots=1$, and $q=l, t$. Hence the formal solutions of (3.25)-(3.28), (3.41) and (3.42) are

$$
U_{n}=-\int_{0}^{2 \pi} \int_{0}^{2 \pi} X_{n}\left(x^{\prime}, \tau^{\prime}\right) G_{l}\left(x, \tau ; x^{\prime}, \tau^{\prime}\right) d x^{\prime} d \tau^{\prime},
$$




$$
V_{n}=-\int_{0}^{2 \pi} \int_{0}^{2 \pi} Y_{n}\left(y^{\prime}, \tau^{\prime}\right) G_{t}\left(y, \tau ; y^{\prime}, \tau^{\prime}\right) d y^{\prime} d \tau^{\prime}, \quad n=1,2,3, \cdots
$$

The reason for (3.44) and (3.45) being formal solutions is the lack of a convergence proof for the infinite series.

4. First iterate $(n=1)$. To obtain the first iterate of $k_{l}^{2}$ and $k_{l}^{2}$, we substitute (3.35) and (3.36) into (3.39) and (3.40) respectively, with $n=1$, and perform the integration to get

$$
\begin{aligned}
& k_{l 1}^{2}=k_{l 0}^{2}-\frac{1}{2 \pi^{2} \epsilon^{2}} \int_{0}^{2 \pi} \int_{0}^{2 \pi} U_{0} p_{1}\left(U_{0}, V_{0}, \cdots\right) d x d \tau, \\
& k_{t 1}^{2}=k_{t 0}^{2}-\frac{1}{2 \pi^{2} \epsilon^{2}} \int_{0}^{2 \pi} \int_{0}^{2 \pi} V_{0} p_{2}\left(U_{0}, V_{0}, \cdots\right) d y d \tau .
\end{aligned}
$$

These equations show that the first correction to $k_{l 0}^{2}$ and $k_{t 0}^{2}$ is due only to the nonlinear part of the equivalent body forces.

Next, upon performing the necessary integration in (3.43)-(3.45) with $n=1$, we obtain

$U_{1}=\frac{\frac{1}{2} \epsilon^{2} \beta_{1} k_{l 1}^{3}}{4\left(k_{30}^{2}-c_{l}^{-2} \omega^{2}\right)-\gamma_{l}} \sin 2(x-\tau)+\frac{1}{2 \pi} \delta^{2} \beta_{2} k_{l_{1}}^{3} \sum_{i=0}^{\infty} \frac{\epsilon_{i}}{k_{l 0}^{2} i^{2}-4 \omega^{2} c_{l}^{-2}-\gamma_{l}}$

. $\left(I_{0 i 1} \sin i x \cos 2 \tau-I_{0 i 2} \sin i x \sin 2 \tau+I_{0 i 3} \cos i x \cos 2 \tau-I_{0 i 4} \cos i x \sin 2 \tau\right)-I_{b}$,

$$
\begin{aligned}
V_{1}=\frac{1}{2 \pi} & \epsilon \beta_{3} k_{t 1} k_{t_{1}}\left[\left(k_{i 1}-k_{t 1}\right) \sum_{i=0}^{\infty} \frac{\epsilon_{i}}{k_{t 0}^{2} i^{2}-\gamma_{t}}\left(I_{0 i 5}^{1} \cos i y+I_{0 i 6}^{1} \sin i y\right)\right. \\
& +\left(k_{t 1}+k_{t 1}\right) \sum_{i=0}^{\infty} \frac{\epsilon_{i}}{k_{t 0}^{2} i^{2}-4 \omega^{2} c_{t}^{-2}-\gamma_{t}}\left(I_{0 i 7}^{1} \cos i y \cos 2 \tau\right. \\
& \left.\left.+I_{0 i 8}^{1} \sin i y \cos 2 \tau-I_{0 i 9}^{1} \cos i y \sin 2 \tau-I_{0 i 10}^{1} \sin i y \sin 2 \tau\right)\right]-I_{t},
\end{aligned}
$$

where

$$
\begin{aligned}
& I_{n i 1}= \begin{cases}\frac{i\left[\sin 2\left(2 \pi \sigma_{n}+\varphi\right)-\sin 2 \varphi\right]}{4 \sigma_{n}^{2}-i^{2}}, & \sigma_{n} \neq \frac{1}{2} i, \\
\pi \cos 2 \varphi, & \sigma_{n}=\frac{1}{2} i,\end{cases} \\
& I_{n i 2}= \begin{cases}\frac{i\left[\cos 2\left(2 \pi \sigma_{n}+\varphi\right)-\cos 2 \varphi\right]}{4 \sigma_{n}^{2}-i^{2}}, & \sigma_{n} \neq \frac{1}{2} i, \\
-\pi \sin 2 \varphi, & \sigma_{n}=\frac{1}{2} i,\end{cases} \\
& I_{n i 3}= \begin{cases}\frac{-2 \sigma_{n}\left[\cos 2\left(2 \pi \sigma_{n}+\varphi\right)-\cos 2 \varphi\right]}{4 \sigma_{n}^{2}-i^{2}}, & \sigma_{n} \neq \frac{1}{2} i, \\
\pi \sin 2 \varphi, \frac{\sigma_{n}}{2}=\frac{1}{2} i,\end{cases} \\
& I_{n i 4}= \begin{cases}\frac{2 \sigma_{n}\left[\sin 2\left(2 \pi \sigma_{n}+\varphi\right)-\sin 2 \varphi\right]}{4 \sigma_{n}^{2}-i^{2}}, & \sigma_{n} \neq \frac{1}{2} i, \\
\pi \cos 2 \varphi, & \sigma_{n}=\frac{1}{2} i,\end{cases}
\end{aligned}
$$




$$
\begin{aligned}
& I_{n i s}^{i}=\frac{-\left(i-j \sigma_{n}^{-1}\right)\left[\cos \left(2 \pi j \sigma_{n}^{-1}-\varphi\right)-\cos \varphi\right]}{\left(1-j \sigma_{n}^{-1}\right)^{2}-i^{2}}, \\
& I_{n i 6}^{i}=\frac{-i\left[\sin \left(2 \pi j \sigma_{n}^{-1}-\varphi\right)+\sin \varphi\right]}{\left(1-j \sigma_{n}^{-1}\right)^{2}-i^{2}}, \\
& I_{n i 7}^{i}=\frac{-\left(1+j \sigma_{n}^{-1}\right)\left[\cos \left(2 \pi j \sigma_{n}^{-1}+\varphi\right)-\cos \varphi\right]}{\left(1+j \sigma_{n}^{-1}\right)^{2}-i^{2}}, \\
& I_{n i 8}^{i}=\frac{i\left[\sin \left(2 \pi j \sigma_{n}^{-1}+\varphi\right)-\sin \varphi\right]}{\left(1+j \sigma_{n}^{-1}\right)^{2}-i^{2}}, \\
& I_{n i \theta}^{i}=\frac{\left(1+j \sigma_{n}^{-1}\right)\left[\sin \left(2 \pi j \sigma_{n}^{-1}+\varphi\right)-\sin \varphi\right]}{\left(1+j \sigma_{n}^{-1}\right)^{2}-i^{2}}, \\
& I_{n i 10}^{i}=\frac{i\left[\cos \left(2 \pi j \sigma_{n}^{-1}+\varphi\right)-\cos \varphi\right]}{\left(1+j \sigma_{n}^{-1}\right)^{2}-i^{2}}, \quad n=0,1,2,3, \cdots, \\
& I_{l}=\int_{0}^{2 \tau} \int_{0}^{2 \tau} G_{l}\left(x, \tau ; x^{\prime}, \tau^{\prime}\right) p_{1}\left[U_{0}\left(x^{\prime}, \tau^{\prime}\right), V_{0}\left(\sigma_{1} x^{\prime}, \tau^{\prime}\right), \cdots\right] d x^{\prime} d \tau^{\prime},
\end{aligned}
$$

and

$$
I_{t}=\int_{0}^{2 \pi} \int_{0}^{2 \pi} G_{t}\left(y, \tau ; y^{\prime}, \tau^{\prime}\right) p_{2}\left[U_{0}\left(\sigma_{1}^{-1} y^{\prime}, \tau^{\prime}\right), V_{0}\left(y^{\prime}, \tau^{\prime}\right), \cdots\right] d y^{\prime} d \tau^{\prime} .
$$

Since $0<\sigma_{n}^{-1}<1$ for all reasonable physical situations, there is no danger that the denominators of $I_{n i 5}^{1}, \cdots, I_{n i 10}^{1}$ are zero.

Let us now collect the above results and reintroduce the original variables $x_{1}$ and $t$. The propagation constants for longitudinal and transverse waves are then

$$
\begin{aligned}
& k_{l}^{2} \sim \frac{\omega^{2}}{c_{l}^{2}}+\gamma_{\imath}-\frac{1}{2 \pi^{2} \epsilon^{2}} \int_{0}^{2 \pi} \int_{0}^{2 \pi} U_{0} p_{1}\left(U_{0}, V_{0}, \cdots\right) d x d \tau, \\
& k_{\imath}^{2} \sim \frac{\omega^{2}}{c_{t}^{2}}+\gamma_{t}-\frac{1}{2 \pi^{2} \delta^{2}} \int_{0}^{2 \pi} \int_{0}^{2 \pi} V_{0} p_{2}\left(U_{0}, V_{0}, \cdots\right) d y d \tau ;
\end{aligned}
$$

the longitudinal wave

$$
\begin{aligned}
u_{1}\left(x_{1}, t\right) & \sim \epsilon \cos \left(k_{l} x_{1}-\omega t\right)+\epsilon^{2} \frac{\beta_{1} k_{l}^{3}}{6 \gamma_{l}} \sin 2\left(k_{l} x_{1}-\omega t\right) \\
+ & \left(\frac{\delta^{2} \beta_{2} k_{l}^{3} k_{l 0}^{2} k_{l 0}^{-2}}{8 \pi\left(4 \omega^{2} c_{l}^{-2}+\gamma_{l}\right)}\left[\cos 2\left(\omega t-2 \pi k_{l 0} k_{t 0}^{-1}-\varphi\right)-\cos 2(\omega t-\varphi)\right]\right. \\
+ & \frac{1}{2 \pi} \delta^{2} \beta_{2} k_{t}^{3} \sum_{\substack{i \times 2 k_{0} k_{l 0}-1 \\
i}}^{\infty} \frac{1}{\left[\omega^{2} c_{l}^{-2}\left(i^{2}-4\right)+\gamma_{l}\left(i^{2}-1\right)\right]\left[4 k_{t 0}^{2} k_{l 0}^{-2}-i^{2}\right]} \\
& \cdot\left\{-\left[\cos 2\left(2 \pi k_{t 0} k_{l 0}^{-1}+\varphi\right)-\cos 2 \varphi\right]\right. \\
& \cdot\left[i \sin i k_{l} x_{1} \sin 2 \omega t+2 k_{t 0} k_{l 0}^{-1} \cos i k_{l} x_{1} \cos 2 \omega t\right] \\
+ & {\left[\sin 2\left(2 \pi k_{t 0} k_{l 0}^{-1}+\varphi\right)-\sin 2 \varphi\right] } \\
& \left.\left.\cdot\left[i \sin i k_{l} x_{1} \cos 2 \omega t-2 k_{t 0} k_{l 0}^{-1} \cos i k_{l} x_{1} \sin 2 \omega t\right]\right\}\right)
\end{aligned}
$$




$$
\begin{aligned}
& +\left[\frac{\delta^{2} \beta_{2} k_{t}^{3}}{2\left(4 \omega^{2} c_{l}^{-2}+\gamma_{l}\right)\left(k_{t 0}^{2} k_{l 0}^{-2}-1\right)+6 \gamma_{l} k_{t 0}^{2} k_{l 0}^{-2}} \sin 2\left(k_{t 0} k_{l 0}^{-1} k_{l} x_{1}-\omega t+\varphi\right)\right] \delta_{i, 2 k_{t 0} k_{l 0}-1} \\
& -I_{l}
\end{aligned}
$$

and the transverse wave

$$
\begin{aligned}
u_{2}\left(x_{1}, t\right) \sim & \delta \cos \left(k_{t} x_{1}-\omega t+\varphi\right) \\
+ & \frac{1}{2 \pi} \epsilon \delta \beta_{3} k_{t} k_{l}\left(\left(k_{t}-k_{l}\right) \sum_{i=0}^{\infty} \frac{\epsilon_{i}}{\left[\omega^{2} c_{t}^{-2} i^{2}+\gamma_{t}\left(i^{2}-1\right)\right]\left[\left(1-k_{l 0} k_{t 0}^{-1}\right)^{2}-i^{2}\right]}\right. \\
& \cdot\left\{\left(1-k_{l 0} k_{t 0}^{-1}\right)\left[\cos \left(2 \pi k_{l 0} k_{t 0}^{-1}-\varphi\right)-\cos \varphi\right] \cos i k_{t} x_{1}\right. \\
+ & \left.i\left[\sin \left(2 \pi k_{l 0} k_{t 0}^{-1}-\varphi\right)+\sin \varphi\right] \sin i k_{t} x_{1}\right\} \\
+ & \frac{\left(k_{t}+k_{l}\right)}{2\left(4 \omega^{2} c_{t}^{-2}+\gamma_{t}\right)\left(1+k_{l 0} k_{t 0}^{-1}\right)}\left[\cos \left(2 \omega t-2 \pi k_{l 0} k_{t 0}^{-1}-\varphi\right)-\cos (2 \omega t-\varphi)\right] \\
- & \left(k_{t}+k_{l}\right) \sum_{i=1}^{\infty} \frac{1}{\left[\omega^{2} c_{t}^{-2}\left(i^{2}-4\right)+\gamma_{t}\left(i^{2}-1\right)\right]\left[\left(1+k_{l 0} k_{t 0}^{-1}\right)^{2}-i^{2}\right]} \\
& \cdot\left\{\left[\cos \left(2 \pi k_{l 0} k_{t 0}^{-1}+\varphi\right)-\cos \varphi\right]\right. \\
& \cdot\left[\left(1+k_{l 0} k_{t 0}^{-1}\right) \cos i k_{t} x_{1} \cos 2 \omega t+i \sin i k_{t} x_{1} \sin 2 \omega t\right] \\
+ & {\left[\sin \left(2 \pi k_{l 0} k_{t 0}^{-1}+\varphi\right)-\sin \varphi\right] } \\
& \left.\left.\cdot\left[\left(1+k_{l 0} k_{t 0}^{-1}\right) \cos i k_{t} x_{1} \sin 2 \omega t-i \sin i k_{t} x_{1} \cos 2 \omega t\right]\right\}\right) \\
- & I_{t} .
\end{aligned}
$$

5. Discussion. The dispersion relations (3.37) and (3.38) for the linearized problem show that the presence of the linear part of the equivalent body forces increases the propagation constants, in turn, decreasing the phase velocities. Eqs. (4.17)-(4.20) essentially consist of the results for the corresponding linearized problem and the corrections from the first iteration. In view of the general mechanism of the iteration scheme, our approximate solutions probably will converge as $n \rightarrow \infty$, if either $\epsilon \ll 1$ and $\delta \ll 1$ or $\beta_{i}(j=1,2,3) \ll 1$. These situations are consistent with the derivation of the approximate equations of motion in Sec. 2 .

From the dispersion relations (4.17) and (4.18), it can be seen that the only corrections to the propagation constants for the linearized values, $k_{\imath 0}$ and $k_{\imath 0}$, come from the nonlinear parts of the equivalent body forces. Because of the positive powers of $\epsilon$ and $\delta$ in these equations, the propagation constants depend upon the amplitudes of the elastic waves and equal the linearized values only when the amplitudes become identically zero. Furthermore, $k_{l}$ and $k_{t}$ will be greater or less than $k_{\imath 0}$ and $k_{t 0}$ according as the signs of

$$
\frac{1}{2 \pi^{2} \epsilon^{2}} \int_{0}^{2 \pi} \int_{0}^{2 \pi} U_{0} p_{1} d x d \tau \text { and } \frac{1}{2 \pi^{2} \delta^{2}} \int_{0}^{2 \pi} \int_{0}^{2 \pi} V_{0} p_{2} d y d \tau
$$

are negative or positive, and therefore the phase velocities $\omega k_{l}^{-1}$ and $\omega k_{t}^{-1}$ are lower or higher accordingly. 
Eq. (4.19) describes the motion of the longitudinal displacement vector approximately. The first term of (4.19) represents the fundamental mode of a propagating wave with phase velocity $\omega k_{l}^{-1}$. The presence of the second term is due to the nonlinear interaction of the fundamental longitudinal wave with itself; it represents a second harmonic propagating with the same phase velocity as that of the fundamental wave. The third and fourth terms come from the nonlinear coupling between the propagating fundamental transverse wave and itself. For $k_{t 0} k_{l 0}^{-1} \neq \frac{1}{2} m, m=3,4,5, \cdots$, the third term gives the space-independent second harmonic oscillation, and the fourth term represents second harmonic standing waves with phase velocities $2 \omega\left(i k_{l}\right)^{-1}, i=1$, $2,3, \cdots$. When $k_{t 0} k_{l 0}^{-1}=\frac{1}{2} m, m=3,4,5, \cdots$, the term with $m=1$ gives a second harmonic propagating wave with phase velocity $\omega\left(k_{t 0} k_{l}\right)^{-1} k_{l 0}$. Finally, the last term represents the sole effect of the nonlinear part of body forces on longitudinal waves; it cannot be discussed in detail without $p_{1}$ being specified explicitly.

The motion of the transverse displacement vector is described by (4.20) approximately. The first term of (4.20) represents the fundamental mode of a propagating transverse wave with phase velocity $\omega k_{t}^{-1}$. The second, third and fourth terms are due to the nonlinear interaction of the fundamental transverse wave with the fundamental longitudinal wave. The second term gives static transverse displacement. The third term is the space-independent second harmonic oscillation, and the fourth term represents second harmonic standing waves with phase velocities $2 \omega\left(i k_{t}\right)^{-1}, i=1,2,3, \cdots$. Finally, the last term represents the sole effect of the nonlinear part of body forces on transverse wave. Note that, so far as the first iteration is concerned, there is no propagating mode other than the fundamental in the transverse displacement vector.

What are the effects of nonlinearity of the elastic medium on propagation constants? What are propagating modes other than the fundamental wave of the transverse displacement vector? These questions will be answered in a subsequent paper by examining the second iterate.

\section{REFERENCES}

[1] D. R. Bland, Nomlinear dynamic elasticity, Blaisdell, Waltham, Mass., 1969

[2] Y. M. Chen and P. L. Christiansen, Application of a modified Newton's iteration method to construct solutions of eigenvalue problems of nonlinear partial differential operators, SIAM J. Appl. Math. 18, 335-345 (1970)

[3] H. B. Keller, Nonlinear bifurcation, J. Differential Equations 7, 417-434 (1970)

[4] L. L. Landau and E. M. Lifshitz, Theory of elasticity, Addison-Wesley, Reading, Mass., 1959

[5] J. B. Keller and L. Ting, Periodic vibrations of syslems governed by nonlinear partial differential equations, Comm. Pure. Appl. Math. 19, 371-420 (1966) 